\title{
$k$-tuple and $k$-tuple total dominations on web graphs
}

\author{
M. P. Dobson, V. Leoni, M. I. Lopez Pujato
}

\begin{abstract}
In this work we address $k$-tuple and $k$-tuple total dominations on the subclass of circular-arc graphs given by web graphs. For the non total version, we present a linear time algorithm based on the regularity of the closed neighborhoods associated with web graphs which allows the use of modular arithmetic for integer numbers. For the total version, we derive bounds for this graph class.
\end{abstract}

\section{Preliminaries}

Domination in graphs is useful in different applications. There exist many variations - such as $k$-tuple domination and $k$-tuple total domination, among others - regarding slight differences in their definitions. These differences make circular-arc graph subclasses adequate and useful mostly due to their relation to "circular" issues, such as in forming sets of representatives, in resource allocation in distributed computing systems,

2000 AMS Subject Classification: 05C69, 05B20, 11A07, 03D15.

Keywords and Phrases: linear algorithm, circular-arc graph subclass, modular arithmetic, bounds.

This research was supported by PICT ANPCyT 0410 (2017-2020) and PID UNR 631 (2018-2022). 
in coding theory [16], and in testing for circular arrangements of genetic molecules 10.

We consider finite simple graphs $G$, where $V(G)$ and $E(G)$ denote its vertex and edge sets, respectively. The open neighborhood of $v \in V(G)$ is $N_{G}(v)=\{u \in V(G): u v \in E(G)\}$ and the closed neighborhood is $N_{G}[v]=N_{G}(v) \cup\{v\}$. The minimum degree of $G$ is denoted by $\delta(G)$ and is the minimum between the cardinalities of $N_{G}(v)$, for all $v \in V(G)$. A graph $G$ is circular-arc if it has an intersection model consisting of arcs on a circle, that is, if there is a one-to-one correspondence between the vertices of $G$ and a family of arcs on a circle such that two distinct vertices are adjacent in $G$ when the corresponding arcs intersect.

Given a graph $G$ and a positive integer $k, D \subseteq V(G)$ is a $k$-tuple (total) dominating set in $G$ if $\left|N_{G}[v] \cap D\right| \geq k\left(\left|N_{G}(v) \cap D\right| \geq k\right)$, for every $v \in V(G)$. When $k \leq \delta(G)+1(k \leq \delta(G)), \gamma_{\times k}(G)\left(\gamma_{k}^{t}(G)\right)$ denotes the cardinality of a $k$-tuple (total) dominating set in $G$ of minimum size. $\gamma_{\times k}(G)\left(\gamma_{k}^{t}(G)\right)$ is called the $k$-tuple (total) dominating number of $G$. Observe that $\gamma_{\times 1}(G)$ is equal to the well-known dominating number $\gamma(G)$; also that $\gamma_{\times k}(G) \leq \gamma_{k}^{t}(G)$ for every graph $G$ and every positive integer $k$. The notions of $k$-tuple domination and $k$-tuple total domination were introduced in [8] and [11], respectively.

For a fixed $k$, the $k$-tuple (total) domination problem is to find in a given graph $G$, a $k$-tuple (total) dominating set in $G$ of size $\gamma_{\times k}(G)\left(\gamma_{k}^{t}(G)\right)$. When the input graph is not connected, it is clear from its definition that the $k$-tuple (total) domination problem can be addressed on each connected component separately to obtain a minimum $k$-tuple (total) dominating set for the entire graph as the union of minimum $k$-tuple (total) dominating sets on each component. Thus, in the sequel we can consider that the input graph is always connected.

The decision problems (fixed $k$ ) associated with these concepts are independently known to be NP-hard [12,13] but polynomial time solvable in some graph classes (see, for example, [2,7, 12,13]). For proper interval graphs, an efficient algorithm for the 1-tuple domination problem is de- 
veloped in [5], but no valid algorithm seems to be currently available for the remaining values of $k$ beyond the one given in [12] for strongly chordal graphs (which is a superclass of proper interval graphs). With a different approach, a polynomial time algorithm was presented recently in 6] for the $k$-tuple total domination problem in proper interval graphs, for each fixed value of $k$.

For general circular-arc graphs — which generalize proper interval graphsefficient algorithms are presented for 1-tuple domination in [5] and for the 2-tuple domination in a recent work [14. Efficient algorithms are known to solve the 1-tuple domination problem [5,9] and the 1-tuple total domination problem [4], but their complexity are not known for any $k$ greater than 1 . It remains challenging to make a breakthrough in the study of the tractability of these problems in subclasses of circular-arc graphs. In this work, we address the subclass given by web graphs. It follows from 16 that web graphs are circular-arc graphs. For a web graph $G$, on the one side the study of $k$-tuple domination (in fact in [1], $k$-tuple domination is referred as $k$-domination) was already started in [1] by means of polyhedral arguments, and solved only for the cases $k=2$ and $k$ being the common degree $\delta(G)$ of the vertices. For the remaining values of $k$, only an upper bound and a lower bound for $\gamma_{\times k}(G)$ were given; these are $k\left\lfloor\frac{n}{\delta(G)+1}\right\rfloor \leq \gamma_{\times k}(G) \leq k\left\lceil\frac{n}{\delta(G)+1}\right\rceil$, where $n=|V(G)|$. On the other side, $k$-tuple total domination on web graphs had not yet been studied.

In this work, the sets of integer numbers, positive integer numbers and non negative integer numbers are denoted respectively by $\mathbb{Z}, \mathbb{Z}^{+}$and $\mathbb{Z}_{0}^{+}$. Besides, every numerical interval is an integer interval, i.e., one of the form $[a, b]$ with $a, b \in \mathbb{Z}$ and $a<b$, together with all integer numbers that are between $a$ and $b$. Given $a, b \in \mathbb{Z}$, the greatest common divisor between $a$ and $b$, i.e., the largest positive integer number that divides both $a$ and $b$, is denoted by $\operatorname{gcd}(a, b)$. 


\section{A vertex set partition related to a web graph}

Given $n, m \in \mathbb{Z}^{+}$with $m \geq 1$ and $n \geq 2 m+1$, a web graph - denoted by $W_{n}^{m}$ - is a graph with $V\left(W_{n}^{m}\right)=\left\{v_{1}, \cdots, v_{n}\right\}$ and $v_{i} v_{j} \in E\left(W_{n}^{m}\right)$ if and only if $j \equiv i \pm l(\bmod n), l \in\{1, \cdots, m\}$ [15. It is clear that $\left|N_{W_{n}^{m}}[v]\right|=2 m+1$ for each $v \in V\left(W_{n}^{m}\right)$. An example is given in Figure 1 .

Let us consider the integer division between $n$ and $2 m+1$ and denote by $c$ the quotient and $r$ the remainder, with $0 \leq r<2 m+1$; i.e., $n=$ $c(2 m+1)+r$. If we denote $\mu:=\operatorname{gcd}(2 m+1, r)$, there exist $l_{1} \in \mathbb{Z}_{0}^{+}$and $l_{2} \in \mathbb{Z}^{+}$such that $r=l_{1} \mu$ and $2 m+1=l_{2} \mu$, and thus $n=\left(c l_{2}+l_{1}\right) \mu$.

Besides, let us denote by $[i]_{\mu}$ for $i, \mu \in \mathbb{Z}^{+}$, the set of all integer numbers that are congruent with $i$ modulo $\mu$. Under this notation and taking into account the fact that $\operatorname{gcd}\left(l_{2}, c l_{2}+l_{1}\right)=1$, we can prove the following lemma and proposition.

Lemma 2.1. The set $S_{i}:=[i]_{\mu} \cap[1, n]$ has cardinality $\frac{n}{\mu}$ for every $i \in$ $\{1, \cdots, \mu\}$.

Example 2.1. For $n=15$ and $m=4$ we have $2 m+1=9, c=1, r=6$ and $\mu=\operatorname{gcd}(9,6)=3$. Sets $S_{i}$ 's are: $S_{1}=[1]_{3} \cap[1,15]=\{1,4,7,10,13\}$, $S_{2}=[2]_{3} \cap[1,15]=\{2,5,8,11,14\}$ and $S_{3}=[3]_{3} \cap[1,15]=\{3,6,9,12,15\}$.

Proposition 2.1. For each $i \in\{1, \cdots, \mu\}$ it holds

$$
S_{i}=\bigcup_{t \in[0, n / \mu-1]}\{w \in[1, n]: w \equiv i+t(2 m+1)(\bmod n)\} .
$$

Proposition 2.1 induces a vertex set ordering of any web graph.

From now on, all sums in the subscripts are taken modulo $n$ and thus, they are in $[1, n]$. Consider a web graph $W_{n}^{m}$, for some $m$ and $n$. To simplify the notation, in the remainder we omit the subscripts in every vertex neighborhood of $W_{n}^{m}$, and thus $N[v]$ indicates $N_{W_{n}^{m}}[v]$.

From the definition of $W_{n}^{m}$, it is clear that $N\left[v_{m+j}\right]=\left\{v_{j}, v_{j+1}, \cdots, v_{j+2 m}\right\}$ (observe this in Figure 1). In the sequel $j$ refers to vertex $v_{m+j}$ of the web 


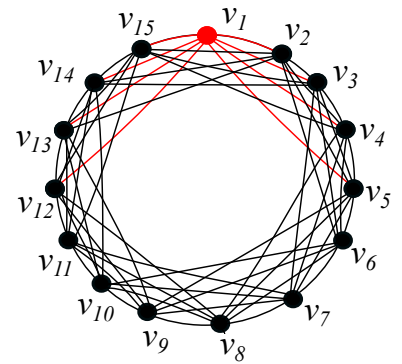

Figure 1: The web graph $W_{15}^{4}$.

graph $W_{n}^{m}$, for $j \in\{1, \cdots, n\}$. For instance in Example 2.1, $S_{1}$ represents the subset $\left\{v_{5}, v_{8}, v_{11}, v_{14}, v_{2}\right\}$ of the vertex set of $W_{15}^{4}$.

As a corollary of Lemma 2.1 and Proposition 2.1, we can state:

Corollary 2.1. $\left\{S_{i}\right\}_{i=1}^{\mu}$ is a partition of $V\left(W_{n}^{m}\right)$ into sets of cardinality $\frac{n}{\mu}$.

\section{A linear time algorithm for $k$-tuple domination}

We first observe that under the notation of the previous section, when $G=W_{n}^{m}$ is a web graph with $n=c(2 m+1)$ (i.e., $\left.r=0\right)$, the upper and lower bounds for $\gamma_{\times k}(G)$ exposed in the preliminaries [1] do coincide and, as a consequence, they derive the exact value of the $k$-tuple domination number, that is $\gamma_{\times k}\left(W_{n}^{m}\right)=k c$ for any $k$ with $1 \leq k \leq 2 m+1$.

The following result shows that sets $S_{i}$ 's have the same number of vertices in each closed neighborhood.

Lemma 3.1. Let $W_{n}^{m}$ be a web graph with $2 m+1=l_{2} \mu$ for $l_{2} \in \mathbb{Z}^{+}$. Then for every vertex $v \in V\left(W_{n}^{m}\right)$ and $i \in\{1, \cdots, \mu\},\left|N[v] \cap S_{i}\right|=l_{2}$.

Proposition 3.1. For every web graph $W_{n}^{m}$, it holds

$$
\gamma_{\times l_{2}}\left(W_{n}^{m}\right)=\frac{n}{\mu}
$$

As mentioned above, Proposition 2.1 leads us to consider an ordering in each $S_{i}$ starting from $i$, in such a way that each element is obtained 


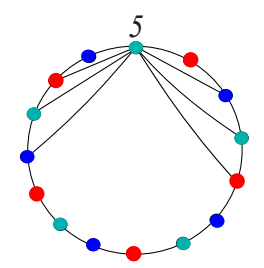

Figure 2: $N[5]$ has $l_{2}=3$ vertices of each $S_{i}$, referring to $W_{15}^{4}$ of Example 2.1.

from the previous one in this ordering as a "circular movement" of $2 m+1$ positions.

Given $W_{n}^{m}, i \in\{1, \cdots \mu\}$ and $j, q$ two vertices in $S_{i}$, we say that $q$ is 1 -contiguous to $j$ when $q \equiv j+2 m+1(\bmod n)$. Observe that if $q$ is 1 contiguous to $j$, then $|N[j] \cup N[q]|$ is equal to $n$ if $c=1$, and to $2(2 m+1)$ if $c \geq 2$. Given $i \in\{1, \cdots, \mu\}$, let us consider in $S_{i}$, the ordering induced by the 1-contiguous relation. To indicate that ordering, we write $\left\langle S_{i}\right\rangle$.

Example 3.1. For $W_{15}^{4}$ (see Example 2.1), we can write $\left\langle S_{1}\right\rangle=(1,10,4,13,7)$, $\left\langle S_{2}\right\rangle=(2,11,5,14,8)$ and $\left\langle S_{3}\right\rangle=(3,12,6,15,9)$.

Given $m, n,\left\langle S_{i}\right\rangle$ for some $i$ and $\alpha<l_{2}$, procedure DOM below returns an $\alpha$-tuple dominating set in $W_{n}^{m}$. In each step, it chooses from $\left\langle S_{i}\right\rangle$ a vertex that is the 1-contiguous vertex to the latest added to $D$ and stops when each vertex of $W_{n}^{m}$ has at least $\alpha$ adjacent vertices that belong to $D$. We take into account Procedure $\mathbf{D I V}(t, w)$ which returns the quotient $(c)$ and the remainder $(r)$ from the Integer Division between two given integer numbers $t$ and $w$. Also, Procedure $\operatorname{PROC}(n, m, i)$ - based on Proposition 2.1 which returns the set $\left\langle S_{i}\right\rangle:=\left(s_{1}^{i}, \cdots, s_{\frac{n}{\mu}}^{i}\right)$.

Procedure DOM $\left(n, m,\left\langle S_{i}\right\rangle, \alpha\right)$

$h=1$

DIV $(n, 2 m+1)$

$\mu=\operatorname{gcd}(2 m+1, r)$

$D=\emptyset$

While $h \leq \alpha$

$$
j=|D|+1
$$




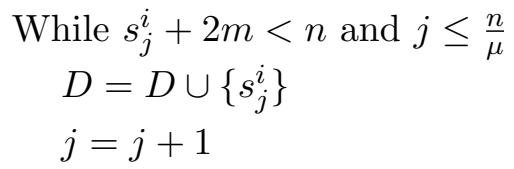

EndWhile

$$
\begin{aligned}
& D=D \cup\left\{s_{j}^{i}\right\} \\
& h=h+1
\end{aligned}
$$

EndWhile

\section{EndProcedure}

Next we present Algorithm 1 based on procedures DOM and PROC. Taking into account Lemma 3.1, when Algorithm 1 adds the entire set $S_{i}$ produced by procedure $\mathbf{P R O C}(n, m, i)$ to $D$, it turns out that each vertex of the input web graph has $l_{2}$ adjacent vertices of $S_{i}$. Depending on the value of $k$, it uses some sets from $S_{2}$ up to $S_{\mu}$ and possibly part of the set $S_{1}$.

ALGORITHM 1 Min $k$-tuple dominating set (fixed $k$ ) of $W_{n}^{m}$

Require: $n, m, k \in \mathbb{Z}^{+}$with $n \geq 2 m+1 \geq k$.

Ensure: A minimum $k$-tuple dominating set $D$ of $W_{n}^{m}$.

$\operatorname{DIV}(n, 2 m+1)$

$\mu:=\operatorname{gcd}(2 m+1, r)$

$\operatorname{DIV}(2 m+1, \mu)$ and print quotient $l_{2}$

$\operatorname{PROC}(n, m, 1)$

If $k \leq l_{2}$ then $D=\operatorname{DOM}\left(n, m,\left\langle S_{1}\right\rangle, k\right)$

Else $\left(k>l_{2}\right)$

DIV $\left(k, l_{2}\right)$ and print quotient $\tilde{c}$ and remainder $\tilde{r}$

If $\tilde{r}=0$ then $D=\operatorname{PROC}(n, m, 1) \cup \cdots \cup \operatorname{PROC}(n, m, \tilde{c})$

Else $D=\operatorname{DOM}\left(n, m,\left\langle S_{1}\right\rangle, \tilde{r}\right) \cup \operatorname{PROC}(n, m, 2) \cup \cdots \cup \operatorname{PROC}(n, m, \tilde{c}+$ 1)

\section{EndIf}

\section{EndIf}

On the one side, ALGORITHM 1 runs in linear (as a function of $n$ ) time. This follows from the facts that $\operatorname{PROC}(n, m, i)$ runs in $O\left(\frac{3 n}{\mu}\right)$-time 
since, to obtain $\left\langle S_{i}\right\rangle$, it starts in vertex $i$ and makes in each step at most three operations (addition of $2 m+1$ to the previous element, comparison with $n$ and possibly subtraction of $n$ ); besides, DOM runs in $O(f(n))$ time, where $f$ is a linear function, since the "While" in each $h$-iteration is performed at most $c(\leq n)$ times and each $h$-iteration is performed at most $\alpha(\leq k \leq 2 m+1 \leq n)$ times.

On the other side, when $D$ is a $k$-tuple dominating set in $W_{n}^{m}$, we have

$$
(2 m+1)|D|=\sum_{v \in D}|N[v]|=\sum_{v \in V\left(W_{n}^{m}\right)}|N[v] \cap D| \geq k n,
$$

thus $\gamma_{\times k}\left(W_{n}^{m}\right) \geq \frac{k n}{2 m+1}$ and moreover, $\gamma_{\times k}\left(W_{n}^{m}\right) \geq\left\lceil\frac{k n}{2 m+1}\right\rceil$. Since ALGORITHM 1 returns a $k$-tuple dominating set in $W_{n}^{m}$ of size exactly $\left\lceil\frac{k n}{2 m+1}\right\rceil$, we conclude that its output is of minimum size.

In all we can state:

Theorem 3.1. For a positive integer $k$ with $k \leq 2 m+1, \boldsymbol{A L G O R I T H M}$ 1 returns in linear time a $k$-tuple dominating set of $W_{n}^{m}$ of size

$$
\gamma_{\times k}\left(W_{n}^{m}\right)=\left\lceil\frac{k n}{2 m+1}\right\rceil .
$$

\section{Bounds for the $k$-tuple total domination num- ber}

As was pointed out in Section $1, \gamma_{\times k}(G) \leq \gamma_{k}^{t}(G)$ for every graph $G$ and positive integer $k$. Nevertheless, the inequality may be strict. For instance, for the complete graph $K_{3}, 2=\gamma_{\times 2}\left(K_{3}\right)<\gamma_{2}^{t}\left(K_{3}\right)=3$.

In a recent work [3], the following upper bound for the total $k$-domination number in terms of the $k$-tuple domination number is given for every graph $G: \gamma_{k}^{t}(G) \leq 2 \gamma_{\times k}(G)-k+1$. Also, the following lower bound is presented in the same work, for every graph $G: \gamma_{k}^{t}(G) \geq\left\lceil\frac{k n-|A|}{\Delta(G)-1}\right\rceil$, where $A$ is the subset of vertices of $G$ with maximum degree $\Delta(G)[3$.

We note that in the case $G$ is a web graph, the set $A$ has cardinality $n$ and $\Delta(G)=2 m$. It is not difficult to prove that the value of $\gamma_{\times k}\left(W_{n}^{m}\right)$ is a tighter lower bound if and only if $1 \leq k \leq \frac{2 m+1}{2}$. 
As a consequence of the work done in the previous sections, we derive the first bounds for the $k$-tuple total domination numbers:

Proposition 4.1. For a web graph $W_{n}^{m}$ with $n \geq 2 m+1$, it holds

- $\gamma_{k}^{t}\left(W_{n}^{m}\right) \leq 2\left\lceil\frac{k n}{2 m+1}\right\rceil-k+1$, for every $1 \leq k \leq 2 m+1$,

- $\left\lceil\frac{k n}{2 m+1}\right\rceil \leq \gamma_{k}^{t}\left(W_{n}^{m}\right)$ for every $1 \leq k \leq \frac{2 m+1}{2}$ and

- $\left\lceil\frac{k n-n}{2 m-1}\right\rceil \leq \gamma_{k}^{t}\left(W_{n}^{m}\right)$ for every $\frac{2 m+1}{2}<k \leq 2 m+1$.

Notice that the lower bound $\left[\frac{k n-n}{2 m-1}\right]$ equals the $(k-1)$-tuple domination number of the web graph $W_{n}^{m-1}$.

\section{References}

[1] G. Argiroffo, M. Escalante, and M. E. Ugarte, On the $k$-dominating set polytope of web graphs, E. Notes in Disc. Math. 36 (2010), 11611168.

[2] G. Argiroffo, V. Leoni, and P. Torres, On the complexity of $\{k\}$ domination and $k$-tuple domination in graphs, Inform. Process. Lett., 115, 6-8 (2015) 556-561.

[3] S. Bermudo, J. C. Hernández-Gómez, and J. M. Sigarreta, On the total k-domination in graphs, Disc. Math. Graph Th., 38 (2018), 301-317.

[4] A. Bertossi, and S. Moretti, Parallel algorithms on circular-arc graphs Inform. Process. Lett., 33, (1989/1990), 275-281.

[5] M.S. Chang, Efficient algorithms for the domination problems on interval and circular-arc graphs, Siam J. Comput. 276 (1998), 16711694. 
[6] N. Chiarelli, T. R. Hartinger, V. A. Leoni, M. I. Lopez Pujato, and M. Milanic, New Algorithms for Weighted k-Domination and Total k-Domination Problems in Proper Interval Graphs, Th. Comp. Sc. 795 (2019), 128-141.

[7] M. P. Dobson, V. Leoni, and G. Nasini, The Limited Packing and Tuple Domination problems in graphs, Inform. Process. Lett. 111 (2011), 1108-1113.

[8] F. Harary, and T.W. Haynes, Double domination in graphs, Ars Combin., 55 (2000) 201-213.

[9] W.L. Hsu, and K.H. Tsai, Linear time algorithms on circular-arc graphs, Inform. Process. Lett. 40, 3 (1991), 123-129.

[10] V. Klee, What are the intersection graphs of arcs in a circle? Amer. Math. Monthly 767 (1969), 810-813.

[11] V.R. Kulli, On n-total domination number in graphs, Graph theory, combinatorics, algorithms, and applications (San Francisco, CA, 1989) (1991), 319-324.

[12] C.S. Liao, and G.J. Chang, k-tuple domination in graphs, Inform. Process. Lett. 87, 1 (2003), 45-50.

[13] D. Pradhan, Algorithm aspects of $k$-tuple total domination in graphs, Inform. Process. Lett.,112 21 (2012), 816-822.

[14] S. Sinha, A. Rana and A. Pa, The 2-Tuple Domination Problem on Circular-arc Graphs, J. of Math. and Inf., 8, (2017), 8, 45-55.

[15] L.E. Trotter, A class of facet producing graphs for vertex packing polyhedra, Discrete Mathematics 12 (1975), 373-388.

[16] A. Tucker, Matrix characterizations of circular-arc graphs, Pacific J. Math. 39.2 (1971), 535-545. 
María Patricia Dobson.

Rosario-Argentina.

Facultad de Ciencias Exactas Ingeniería y Agrimensura. Universidad Nacional de Rosario. pdobson@fceia.unr.edu.ar

Valeria Alejandra Leoni.

Rosario-Argentina.

Facultad de Ciencias Exactas Ingeniería y Agrimensura. Universidad Nacional de Rosario. Consejo Nacional de Investigaciones Cientícas y Técnicas.

valeoni@fceia.unr.edu.ar

María Inés Lopez Pujato.

Rosario-Argentina.

Facultad de Ciencias Exactas Ingeniería y Agrimensura. Universidad Nacional de Rosario. Consejo Nacional de Investigaciones Cientícas y Técnicas.

lpujato@fceia.unr.edu.ar 